\title{
Prevalence Rate of Disruptive Behavior Disorders among the
}

\section{Preschool Children}

\author{
MojtabaHabibi $^{1}$, NargesZamani ${ }^{2}$, Mani B.Monajemi ${ }^{3}$, Zahra Fadaei ${ }^{4}$
}

\section{ABSTRACT}

Background:Disruptive BehaviorDisorders (Conduct Disorder, Oppositional Defiant Disorder, and Attention Deficit Hyperactivity Disorder) are one of the most common psychiatric disorders among children. Due to high prevalence of this disorder and its destructive effects on individual's general functioning, the Prevalence Rate of Disruptive BehaviorDisorderamong the preschool children in Hamedan city was assessed throughout this study.

Method:602 preschool children were selected via random cluster sampling method. Data of this cross-sectional research has been collected through complied questionnaire based on the DSM-5 Disruptive BehaviorDisorder symptoms and CSI-4 test. The data of this investigation has been analyzed and evaluated via SPSS software.

Results: The results showed higher prevalence of Disruptive Behavior disorders (Hyperactivity and Impulsive Disorder (13.78), Attention Deficit disorder (11.79), both Attention Deficit Hyperactivity Disorder (9.63), Oppositional Defiant Disorder(5.31) and Conduct Disorder (4.48)) in boys in comparing to girls.

Conclusion:Among all sub-types of Disruptive Behavior Disorders, ADHD had the highest prevalence among the Children.Due to the high prevalence of ADHD among primary school students and its adverse outcomes, following proper therapeutic/Interventional procedures is advised.

Keywords:Disruptive BehaviorDisorder, Conduct Disorder, Oppositional Defiant Disorder, Attention Deficit Hyperactivity Disorder.

In some cases,one aspect of child psychopathology may be demonstrative of climax of specific trajectory in comparing to normal range of that aspect in a normal child (Sartorius et al., 2008). There is fine Border between normal and abnormal, which has not been precisely defined. Although, sometimes cutting point may be exact and demonstrative (Zamani, Habibi, 2012).

\footnotetext{
${ }^{1}$ Family Therapy Department, Institute, Shahid Beheshti University, Tehran, Iran, (Email: mo_habibi@sbu.ac.ir) ${ }^{2}$ Research Center and Department of Social Medicine, Faculty of Medicine, Ilam University of Medical Sciences, Ilam, Iran

${ }^{3}$ Department of Clinical Psychology, University of Tehran, Tehran, Iran, (Email:mani.b.monajemi@warwickgrad.net,mani.b.monajemi@ut.ac.ir)

${ }^{4}$ Family Research Institute, ShahidBeheshti University, G. C., Iran

(c) 2015 I M Habibi, N Zamani, M. B Monajemi, Z Fadaej; licensee IJIP. This is an Open Access Research distributed under the terms of the Creative Commons Attribution License (http://creativecommons.org/licenses/by/2.0), which permits unrestricted use, distribution, and reproduction in any Medium, provided the original work is properly cited.
} 


\section{Prevalence Rate of Disruptive Behavior Disorders among the Preschool Children}

Major classifications of children psychiatric disorders are: Developmental disorders, Emotional problems or internalization, Disruptive behavior disorders or Externalization (Sartorius et al., 2008).The 1964 UK Isle of Wight epidemiological survey showed that approximately $7 \%$ of children suffer from at least one type of psychiatric disorders in mid-childhood (Ostberg, 1998). This number proved to be lower than actual number and the actual prevalence is two times bigger than aforementioned number (Crinc, Greenburg, 1997). Disruptive behavior disorders are prevalent and disabling disorders, which cause problems for teachers, parents and even children themselves. These disorders are accompanied by so many complications such as social problems; most of the times they are diagnosed in early period of primary school. Furthermore, these disorders affect the child's educational process and they will elevate the risk of mental problems in adulthood in these children (Najafi et al., 2009). Conduct Disorder (CD), Oppositional Defiant Disorder (ODD) and Attention Deficit Hyperactivity Disorder (ADHD) can mentioned as disruptive behavior disorders or externalization (Sartorius et al., 2008).Adverse consequences of these disorders are not limited to period of childhood and they also may endanger the future life of these children and their families.

Conduct Disorder (CD) is characterized by a repetitive and persistent pattern of behavior in which the basic rights of others or major age-appropriate societal norms or rules are violated, as manifested by the presence of three (or more) of the following criteria in the past 12 months, with at least one criterion present in the past 6 months(Sadock et al., 2006):

Aggression to people and animals: (1)often bullies, threatens, or intimidates others (2) often initiates physical fights (3) has used a weapon that can cause serious physical harm to others (e.g., a bat, brick, broken bottle, knife, gun) (4) has been physically cruel to people (5) has been physically cruel to animals (6) has stolen while confronting a victim (e.g., mugging, purse snatching, extortion, armed robbery) (7) has forced someone into sexual activity

Destruction of property: (1) has deliberately engaged in fire setting with the intention of causing serious damage(2) has deliberately destroyed others' property (other than by fire setting)

Deceitfulness or theft: (1) Has broken into someone else's house, building, or car(2) Often lies to obtain goods or favors or to avoid obligations (i.e., "cons" others)(3)Has stolen items of nontrivial value without confronting a victim (e.g., shoplifting, but without breaking and entering; forgery)

Serious violations of rules: (1) often stays out at night despite parental prohibitions, beginning before age 13 years (2) has run away from home overnight at least twice while living in parental or parental surrogate home (or once without returning for a lengthy period) (3) is often truant from school, beginning before age 13 years.

The disturbance in behavior causes clinically significant impairment in social, academic, or occupational functioning(Halgin, Whitborne, 2002). 


\section{Prevalence Rate of Disruptive Behavior Disorders among the Preschool Children}

According to DSM-1V, existence of three out five behaviors is essential for diagnosis: 1. Aggression and bullying 2.Threatening 3) Intimidating others 4) Staying out at night despite parental prohibitions 5) Onset before 13 (APA, 2008).

Prevalence of this disorder in general population has been estimated between $1-10 \%$ and it is 412 times more prevalent among boys (Sadock et al., 2006). These children maintain higher tendency toward Alcohol dependency and antisocial personality disorder in their adulthood (APA, 2008).

Oppositional Defiant Disorder (ODD) is one of the most common reasons of children's reference to mental health centers. A lot of children, who demonstrate negative/defiant behavior, tend to find different ways in order to express themselves in their adulthood (Sadock et al., 2006). In ODD burst of anger in child, constant disobey from authority figure are beyond expectation in comparing to normal children. ODD can be identified as, persistent pattern of anger, irritability, arguing, defiance or vindictiveness toward you and other authority figures without violating any serious social norms (Halgin, Whitborne, 2002). Epidemiological studies of non-clinical samples showed that these behaviors could be noticed in $16 \%$ to $2 \%$ of school kids and its more prevalent between boys in comparing to girls (Sadock, Sadock, 2005).

Attention Deficit Hyperactivity Disorder (ADHD) is one of the most common neurodevelopment disorders among children that may persist even till adulthood and it can disturb individual's social life, educational process and it may cause a lot of family disturbances (Barkley, 2010).ADHD, similar to hyperkinetic disorder in the ICD-10 is a developmental neuropsychiatric in which there are significant problems with executive functions (e.g., attentional control and inhibitory control) that cause attention deficits, hyperactivity, or impulsiveness which is not appropriate for a person's age (Sadock, Sadock, 2007). In past it was assumed that hyperactivity is main disabling symptoms of this disorder; nowadays scientists believe that hyperactivity is secondary symptom of impulse control deficit (APA, 2008). Today in diagnosis criteria of ADHD, hyperactivity and impulsivity share common aspect(Barkley, 1997).In the United States, thiscriteriais defined by the American Psychiatric Association in the DSM. Based on the DSM criteria, there are three sub-types of ADHD:

1. ADHD mainly inattentive type presents with symptoms including being easily distracted, forgetful, daydreaming, disorganization, poor concentration, and difficulty completing tasks.

2. ADHD, predominantly hyperactive-impulsive type presents with excessive fidgetiness and restlessness, hyperactivity, difficulty waiting and remaining seated, immature behavior; destructive behaviors may also be present.

3. ADHD, combined type is a combination of the first two subtypes.

Regarding diagnosis, onset of some of the symptoms should be before 7 years old, however in most cases diagnosis is being made after 7 years old, when this disorder has disrupted the individual's life already (Closson, 2010).In early 1900, this disorder was defined as impulsive children who suffered from neurocognitive damages as encephalitis and it was categorized under 


\section{Prevalence Rate of Disruptive Behavior Disorders among the Preschool Children}

Hyperactivity syndrome (Conners, 1970).In 1960, heterogeneous group of children afflicted by Akathisia, learning disability and emotional instability without any neurological problems and with minimum brain damage were discussed, after that various hypotheses were discussed regarding this disorder (Sadock, Sadock, 2007). Epidemiological studies in United States showed that $2 \%$ to $20 \%$ of primary school children are under diagnosis of this disorder (Tamm et al., 2005) and approximately 3\% to $7 \%$ of this number is diagnosed before puberty. Furthermore, prevalence rate is higher as $1 / 2$ in boys and 1/9 in girls (Bussing et al., 2010).

Since behavioral problems influence all personal/social aspects of afflicted individuals; hence, assessing and identifying various aspects of this disorder inchildren in order to implement proper prevention/intervention procedure seems to be vital. According to various studies, behavioral problems may persist and even worsen as the child ages. Thus, precise and impeccable intervention plays a substantial role in dealing with these problems. Main goal of this study was to assess the behavioral problems of preschool children in Hamedan City. Hopefully the results may help the clinicians to intervene and implement suitable therapeutic approach in a process of dealing with these disabling disorders.

\section{MATERIALS AND METHODS}

Current study is cross-sectional research. Statistical society of this study included all preschool children of Hamedan city during 2013-2014. Current sample included 602 individuals (301 boys, 301 girls), who were chosen via cluster sampling method. Regarding analyzing data, descriptive statistics methods (percentage, frequency) and for comparing gender's proportions ( $\mathrm{Z}$ test) were used. Data of this cross-sectional research have been collected through complied questionnaire based on the DSM-5 Disruptive BehaviorDisorder symptoms and CSI-4 (Parent Checklist) test. The Child Symptom Inventory-4 (CSI-4) is a behavior rating scale that screens for DSM-V emotional and behavioral disorders in children between 5 and 12 years old. Parent Checklist contains 112 questions and 41 of it are about A, B, C groups which assess ADHD, CD and ODD. Details of group are as explained below:

Group A: ADHD; Attention deficiency sub-type (9 questions), Impulsivity sub-type(9 questions), combined sub-type (18 questions)

Group B:ODD (8 questions)

Group C:CD (15 questions)(MohamadEsmail, 2001).

Child Symptoms Inventory has been assessed in different studies and its validity, reliability and accuracy have been studied. In a study conducted by Grayson and Carlson (Grayson, Carlson, 1991), accuracy of CSI-3R with respect to ODD, CD and ADHD were reported as 93\%, 93\% and 77\% respectively. Other studies reported correlation of CSI-3R between ODD, CD and ADHD as 66\%, 58\% and 72\% respectively (Gadow, Sprafkin, 1994). In Kalantari and Colleagues study (Neshatoost et al., 2008), validity of this inventory via Bi-Section algorithm was assessed and the result for Parent Checklist and Teacher Checklist estimated to be $91 \%$ and 85\% respectively.In Mohammad Esmail’s study (2001), 9 psychiatrists confirmed content related 
validity of CSI-4. Based on Tavakoli and Colleagues 'study (Tavakoli et al., 2005), reliability of Parent Checklist via retest reliability was $90 \%$. In Najafi and Colleagues' study, reliability of this checklist was via Cronbach’s Alpha was estimated as $90 \%$.

\section{RESULTS}

Table No1 Prevalence of behavioral disorders in sample group

\begin{tabular}{|ll|}
\hline Disorder & Frequency (Percentage) \\
\hline Attention Deficit & $71(11 / 79)$ \\
\hline Hyperactivity- Impulsivity & $83(13 / 78)$ \\
\hline Combined & $58(9 / 63)$ \\
\hline ODD & $32(5 / 31)$ \\
\hline CD & $27(4 / 48)$ \\
\hline
\end{tabular}

Data on Table No1 show the prevalence of Attention Deficit (11/79), Hyperactivity- Impulsivity (13/78), Combined (9/63), ODD (5/31) and CD (4/48).

Table No2 is demonstrating prevalence of behavioral disorders in sample groupwith respect to severity of disorder.

Table No2 Prevalence of behavioral disorders with respect to severity of disorder

\begin{tabular}{|c|c|c|c|c|c|c|c|c|c|c|}
\hline \multirow{2}{*}{$\begin{array}{c}\text { Type of } \\
\text { Disorder }\end{array}$} & \multicolumn{2}{|c|}{ Severity } & \multicolumn{2}{|c|}{ Mild-Severe } & \multicolumn{2}{|c|}{ Beyond Severe } & \multirow{2}{*}{$\begin{array}{c}\text { Total } \\
\text { Percenta } \\
\text { ge }\end{array}$} & \multirow[t]{2}{*}{ Mean } & \multirow[t]{2}{*}{ SD } & \multirow[t]{2}{*}{$\mathbf{Z}$} \\
\hline & Gender & Frequency & Percentage & Frequency & Percentage & $\begin{array}{l}\text { Frequen } \\
\text { cy }\end{array}$ & & & & \\
\hline \multirow{2}{*}{$\begin{array}{c}\text { Attention } \\
\text { Deficit }\end{array}$} & Boy & 37 & $6 / 14$ & 53 & $8 / 81$ & 90 & $14 / 95$ & $6 / 46$ & $5 / 38$ & \multirow[t]{2}{*}{$5 / 98$} \\
\hline & Girl & 19 & $3 / 15$ & 33 & $10 / 13$ & 52 & $8 / 64$ & $5 / 37$ & $4 / 15$ & \\
\hline \multirow{2}{*}{$\begin{array}{l}\text { Hyperactiv } \\
\text { ity } \\
\text { Impulsivity }\end{array}$} & Boy & 53 & $8 / 81$ & 43 & $7 / 14$ & 96 & $15 / 95$ & $5 / 95$ & $3 / 01$ & \multirow[t]{2}{*}{$5 / 85$} \\
\hline & Girl & 28 & $4 / 66$ & 31 & $5 / 15$ & 59 & $9 / 80$ & $4 / 41$ & $6 / 46$ & \\
\hline \multirow[t]{2}{*}{ Combined } & Boy & 61 & $5 / 48$ & 39 & $6 / 48$ & 100 & $16 / 62$ & $9 / 46$ & $8 / 26$ & \multirow[t]{2}{*}{$4 / 84$} \\
\hline & Girl & 33 & $10 / 13$ & 23 & $3 / 82$ & 55 & $9 / 13$ & $6 / 36$ & $6 / 41$ & \\
\hline \multirow[t]{2}{*}{ ODD } & Boy & 23 & $3 / 82$ & 30 & 4/99 & 53 & $8 / 80$ & $5 / 17$ & $5 / 32$ & \multirow[t]{2}{*}{$5 / 86$} \\
\hline & Girl & 15 & $2 / 49$ & 20 & $3 / 33$ & 35 & $5 / 82$ & $4 / 85$ & $4 / 25$ & \\
\hline \multirow[t]{2}{*}{ CD } & Boy & 30 & $4 / 99$ & 28 & $4 / 66$ & 58 & $9 / 63$ & $5 / 78$ & $5 / 24$ & \multirow[t]{2}{*}{$4 / 68$} \\
\hline & Girl & 19 & $3 / 15$ & 15 & $2 / 49$ & 34 & $5 / 65$ & $4 / 31$ & $4 / 02$ & \\
\hline
\end{tabular}

According toTable No2,regardingAttention Deficit Z was 5/98; it means thatAttention Deficit is more prevalent among boys. With respect to Hyperactivity-Impulsivity Z estimated as 5/85. It again means thatHyperactivity-Impulsivityis more prevalent among boys. As same as Attention Deficit and Hyperactivity Impulsivity types, regarding Combined type $(Z=4 / 84)$ boys have higher tendency in comparing to girls. Viewing $\mathrm{ODD}(\mathrm{Z}=5 / 86)$, meaningful difference is noticed between prevalence of this disorder with respect to gender. It means that boys are more prone to ODD. Considering CD Z (4/68), meaningful difference is noticed betweenproportions of this 


\section{Prevalence Rate of Disruptive Behavior Disorders among the Preschool Children}

disorder with respect to gender. It means that boys maintain higher prevalence rate toward CD in comparing to girls.

\section{DISCUSSION AND CONCLUSION:}

Current study was conducted in order to assess prevalence of disruptive behavior disorder (CD, ODD and ADHD) among preschool children. General prevalence of behavioral disorders estimated as 42/01 in a range of mild to severe. Based on Carr's (Carr, 1999) researches, higher percentages of these disordersare noticed in urban societies rather than rural and western cultures rather than eastern. Based on recent findings, most prevalent behavioral disorders were: Hyperactivity-Impulsivity (13/78), Attention Deficiency (11/79), Combined type (9/63), ODD (5/31) and CD (4.48). General prevalence of ADHD in current study via CSI-4 estimated as 5/95 in boys and 4/41 in girls. Analysis of data showed that there is significant difference between boys and girls regarding Attention deficit disorder and it was more prevalent among boys and this result is consistent with the results of the studies conducted by Bussing and Colleagues (Bussing et al., 2010),Sadock and Sadock(2007), Halgin and Whitborne(2002) and American Psychiatric Association(2000).

For diagnosis, onset of some of the symptoms should be before 7 years old and sometimes parents can't spot this disorder till primary school (Sadock, Sadock, 2005) and teachers notice this disorder by noticing problematic learning function of the child. These children may have lower score in educational progress tests in comparing to normal children. In parents of these children, rate of hyperkinesia, antisocial behavior, alcohol dependency and substance abuse is higher comparing to parents of normal children (Sadock, Sadock, 2007). In current study, ODD was more prevalent among boys and this result is consistent with the results of the studies conducted by Cohen and Colleagues (1993), Sadock and Sadock(2007), Halgin and Whitborne(2002) and American Psychiatric Association(2000). General prevalence ofCD is in a range of $2-10 \%$ and it may differ based on different methods of collecting data. Proportion between male and female may differ as 1-3 or 1-5 based on age of individuals. Result of current study is consistent with the results of the study conducted by Sadock and Sadock(2007), Halgin and Whitborne(2002). American Psychiatric Association (2000), Anderson and Colleagues(1987), Fombonee(1994) and Carr(1999).

General findings of this study about higher prevalence of these disorders in boys in comparing to girls are consistent with the results of the studies conducted by McGoey and Colleagues (2002), Whitmore and Colleagues (1997) and McDermott (1996). In their studies they discussed role of educational level of family in mental disorders. Higher prevalence of behavioral disorders in boys may be due to higher biological vulnerability of boys in comparing to girls. Since prenatal period, boys are much more prone to prenatal death and it seems that serious diseases, inappropriate diet and nutrition harm boys more than girls. 
Because ofsmall sample size of current study, generalization of the results to other cities or nations with different ethological/cultural/educational backgrounds is inappropriate and generalization should be followed precisely. The prevalence of behavioral disorders among preschool/school children should be studied more and clinicians, teachers and parents should elevate their awareness regarding this spectrum of disorders in order to be able to faicilitateimpeccable interventional procedures.

\section{Authors' contributions}

MHand NZ conceived and designed the evaluation and they conducted whole process of this study. MBM and ZF collected and interpreted the clinical data and drafted the manuscript.All authors read and approved the final manuscript.

\section{Acknowledgements}

We would like to thank all patients who participated in the study.

\section{Declaration of interest}

None declared

\section{REFERENCE:}

American Psychiatric Association.Diagnostic and statistical manualofmental disorder. (3rd edn_revised).Washington DC: Author; 2000.

Anderson J MR. "DSM-III disorders in preadolescent children. Archives of General psychiatry1987; 44: 69-76.

Barkley R A. Attention-Deficit Hyperactivity Disorder. New York:Guilford Press; 2006.

Barkley R A. Behavioral inhibition, sustained attention, and executive functions: Constructing a unifying theory of ADHD. Psychological Bulletin 1997; 121:65-94.

Bussing R, Mason DM, Bell L, Porter PH, Garvan C. Adolescent out com of childhood attention deficit/hyperactivity disorder in a diverse Community sample .Journal of American Academy of Child and Adolscent Psychiatry 2010; 49 (6):565-605.

Carr A. The Handbook of Child and AdolescentClinical Psychology.A Contextual Approach.London: Routledge; 1999.

Closson M S. Investigating the role of executive function in social decision-making in children with ADHD. A thesis submitted in partial fulfillment of the requirements for the degree of Doctor of Philosophy, HofstraUniversity 2010.

Cohen P, Kasen j \& Carmen N. Epidemiological study of disorders in late childhood and adolescence, age and gender- specific prevalence. Journal of Child Psychology, Psychiatric and Allied Diseiplines1993; 34 (6): 851-67.

ConnersCK .Symptom patterns in hyperkinetic, neurotic and normal children. Child Development1970; 41:667-682. 


\section{Prevalence Rate of Disruptive Behavior Disorders among the Preschool Children}

Crinc K, Greenberg MT. Minor parenting stresses with young children. Child development1997; 54:209-217

Eglund N, HansenKF. Behavioral disorders in Danish schools: A quantitative survey. European Journal of Special Needs Education 2000; 15:158-170.

Fombonne E. The child behaviour checklist and the Rutter Questionnaire.Journal of Child Psychology and Psychiatry1994; 19:32-34.

Gadow KD, Sprafkin J. Child symptom inventories manual. Story Brook, NY: checkmate Plus 1994.

Grayson P, Carlson GA. The utility of a DSM-III-R based checklist in screeningchildpsychiatryic patients Journal of the American Academy of child and Adolescents Psychiatry 1991; 30: 69-673.

Halgin RP, Whitborne SK. Abnormal Psychology: Clinical Psychological Disorder 4th ed. New Yourk, McGraw-Hill; 2002.

McDermott A. Nationwide study of developmental and gender prevalence for psychopathology in child and adolescence. Journal of Abnormal Child Psychology1996; 2: 53-60.

McGoey KE, Eckert TL and DuPaul G J. Early intervention for preschool-age children with ADHD: A literature review. Journal of Emotional Behavioral Disorders 2002; 10: 14-29.

MohamadEsmail E. Evaluation ofreliability, validity, and determinecut pointsCSI4disordersonprimary and junior secondaryschoolstudents6-14years oldTehran. Tehran: Institute for Special Children; 2001. (Persian).

Montoya A, ColomF, Ferrin M. Is psycho educational for parents and teachers ofchildren and adolescent with ADHD efficacious? A systematic literature review: European psychiatry 2011:166-175.

Najafi M, Foladchang M, Alizade H, Mohammadifar MA. Epidemiology of ADHD, Conduct, Oppositional defiant in shiraz students. Research on Exceptional Children 2009;9 (3): 239-254.

Neshatoost M, Klantari M, Yoosefi AR. Prevalence ofhyperactivity disorderinfirst and secondgrade studentsof elementary schoolsin Isfahan. Journalof EducationalandPsychologicalResearchIsfahan University 1(1): (Persian).

Ostberg M. Parental stress, psychosocial problems, and responsiveness in helpseeking parents with small (2-45 months old) children. ActaPeadiatrica1998; 87: 69-76.

Sadock BJ, Sadock VA. Comprehensive textbook of psychiatry. York, USA: Lippincott Williams \& Wilkins; 2007.

Sadock BJ, Sadock VA. Comprehensive textbook of psychiatry. York, USA: Lippincott Williams \& Wilkins2005.

Sartorius N, Leff J, Lopez J, Ibor L, Maj M, Okasha A. Family \& Mental Disorders. RC, 2008.

Tamm L, Swanson JM, Lerner M A, Childress C, Patterson B, Lakes K, et al. Intervention for preschoolers at risk for Attention Deficit /Hyperactivity Disorder (ADHD) Service before diagnosis. Clinical Neuroscience Research 2005; 5: 247-253. 
Tavakoli J, Bolhari J, Mahriar AH, Dozhkam M.The epidemiology ofbehavior disordersand attentiondeficit harassmentin school childrenGhonabad.Mentality \& behavior journal.3 (9): 10 (Persian).

Whitmore HA, Mikulich S \& Thompson KT. Influences an adolescence: Conduct disorder, depression, attention deficit hyperactivity disorder”. Drug and Alcohol Dependence1997; 4: 87-97.

Zamani N, Habibi M. comparing the influence of both dialectic and cognitive behavior therapies to maintain mothers' mental health whose childrenwith Special needs. Journal of Research \& Health Social Development \& Health Promotion Research Center. 1392.123. T.27984. (Persian). 